University of Nebraska - Lincoln

DigitalCommons@University of Nebraska - Lincoln

$9-1-1996$

\title{
Film Stress and Domain Wall Pinning in Sesquilayer Iron Films on $\mathrm{W}(110)$
}

D. Sander

Max-Planck-Institut für Mikrostrukturphysik

Ralph Skomski

University of Nebraska-Lincoln, rskomski2@unl.edu

C. Schmidthals

Max-Planck-Institut für Mikrostrukturphysik

Axel Enders

Max-Planck-Institut für Mikrostrukturphysik, a.enders@me.com

J. Kirschner

Max-Planck-Institut für Mikrostrukturphysik

Follow this and additional works at: https://digitalcommons.unl.edu/physicsskomski

Part of the Physics Commons

Sander, D.; Skomski, Ralph; Schmidthals, C.; Enders, Axel; and Kirschner, J., "Film Stress and Domain Wall Pinning in Sesquilayer Iron Films on W(110)" (1996). Ralph Skomski Publications. 30.

https://digitalcommons.unl.edu/physicsskomski/30

This Article is brought to you for free and open access by the Research Papers in Physics and Astronomy at DigitalCommons@University of Nebraska - Lincoln. It has been accepted for inclusion in Ralph Skomski Publications by an authorized administrator of DigitalCommons@University of Nebraska - Lincoln. 


\title{
Film Stress and Domain Wall Pinning in Sesquilayer Iron Films on W(110)
}

\author{
D. Sander, R. Skomski, C. Schmidthals, A. Enders, and J. Kirschner \\ Max-Planck-Institut für Mikrostrukturphysik, Weinberg 2, 06120 Halle, Germany
}

(Received 12 March 1996)

\begin{abstract}
We present an in situ investigation of the correlation between elastic and magnetic properties of monolayer iron films on W(110). Sesquilayers, consisting of two-monolayer patches on a nearly ideal monolayer film, exhibit anomalous elastic properties and a strikingly high in-plane coercivity of order $0.3 \mathrm{~T}$. The sesquilayer coercivity maximum is explained by a novel domain wall pinning mechanism, based on an enhanced exchange interaction in the two monolayer thick patches. This rather unique behavior is restricted to (110) surfaces but does not occur on (100) and (111) thin films. [S0031-9007(96)01136-2]
\end{abstract}

PACS numbers: 75.50.-y, 75.70.-i, 81.15.Tv

The heteroepitaxy of $\mathrm{Fe}$ on $\mathrm{W}(110)$ is governed mainly by the large lattice mismatch $f=9.4 \%$ derived from the elemental lattice constants $a_{\mathrm{W}}=3.165 \AA$ and $a_{\mathrm{Fe}}=$ $2.866 \AA[1]$. As a consequence of this mismatch only the first monolayer (ML) [2] iron grows pseudomorphically on $\mathrm{W}(110)$ at $300 \mathrm{~K}$ [3]. Misfit dislocations caused by the reduction of the strain energy of the $\mathrm{Fe}$ film are already created in islands of the second monolayer at a Fe coverage of about 1.5 pseudomorphic monolayers, as shown in a recent scanning tunneling microscopy (STM) study [4]. A central-force model [5] involving bulk iron elastic constants yields an elastic energy per surface atom of order $0.3 \mathrm{eV}$, which is a formidable contribution to any energy consideration regarding growth, structure, and magnetism of the iron film. Thus, stress induced effects are likely to affect the behavior of the film and will be discussed in this Letter.

Not only the morphology of the film undergoes a transition from 1 to 2 ML thickness [4], but also four different magnetic regimes are of interest: (i) a submonolayer region, paramagnetic due to the absence of magnetic percolation [6], (ii) a ferromagnetic one monolayer region, characterized by a pronounced twofold in-plane anisotropy [7], (iii) an intermediate, "sesquilayer" region, consisting of second layer islands on top of a one monolayer sea exhibiting, reportedly [8], antiferromagnetic order, and (iv) a $2 \mathrm{ML}$ region without striking magnetic properties. The subject of this work is the investigation of the sesquilayer region at a coverage of $1.5 \mathrm{ML}$. We present for the first time stress measurements with submonolayer sensitivity taken during the growth of ultrathin iron films on W(110). The magnetism of the sesquilayer films is investigated by in situ Kerr effect measurements; the observed coercivity is explained by a novel domain wall pinning mechanism which is estimated to be 10 times stronger than the stress induced coercivity increase.

The iron films were grown under ultrahigh vacuum (UHV) conditions on clean W(110) substrates at $300 \mathrm{~K}$. Film and sample cleanliness were checked by Auger electron spectroscopy, the contamination level due to the only contaminants oxygen and carbon was found to be less than
$1 \%$ of a monolayer. Iron was evaporated by $e$-beam evaporation of a thoroughly outgassed high-purity iron wire at a rate of $1 \AA / \mathrm{min}$, the deposited amount of iron was controlled by an integrated flux monitor. During growth, the sample curvature and thus the stress in the film were monitored using an optical beam deflection technique as described earlier [9]. In short, we use a $0.15 \mathrm{~mm}$ thin $\mathrm{W}(110)$ crystal that is clamped at its top end, so that the crystal is free to bend due to film stress on its front surface. By reflecting a laser beam from the bottom end of the crystal to a split photodiode, we obtain a position signal that is converted to the film stress. Film thickness was checked by Auger electron spectroscopy and by calibrating the iron evaporator against a thickness monitor using a quartz oscillator. The magnetic analysis of the iron films was done in situ by magneto-optic Kerr effect (MOKE) measurements [10]. Using an external electromagnet with a yoke penetrating the UHV chamber, MOKE measurements could be performed in fields of up to $0.4 \mathrm{~T}$. Our in situ stress and Kerr measurements are supplemented by STM experiments done in a second vacuum chamber, to supply us with the structural information on the iron film.

Figure 1 shows the film stress as a function of the iron coverage. Quite surprisingly, the deposition of up to $0.7 \mathrm{ML}$ of iron leads to a negative position signal, indicating compressive stress on the sample surface. The minimum of the stress curve corresponds to a strikingly large compressive stress of $-5 \mathrm{~N} / \mathrm{m}$. Assigning this value to an average film thickness of $0.7 d_{(110)}$, where the (110)layer thickness $d_{(110)}=a_{\mathrm{Fe}} / \sqrt{2}$, results in a film stress of $35 \mathrm{GPa}$, which is far beyond the elasticity limit of high-strength materials such as $\mathrm{CrNi}$ steel [11]. From the lattice mismatch between iron and tungsten, a tensile stress is expected, as the Fe film grows pseudomorphically, thus strained by $10.4 \%$. For increasing coverage, a tensile stress sets in. First, the stress increase is of the order of $4 \mathrm{~N} / \mathrm{m}$ per monolayer, then at $1.5 \mathrm{ML}$ coverage a kink is observed after which the stress increase is lowered to $2.2 \mathrm{~N} / \mathrm{m}$ per monolayer. At $300 \mathrm{~K}$, no stress relaxation was observed. Interrupting the growth process at any point freezes the stress at the respective value of the stress 


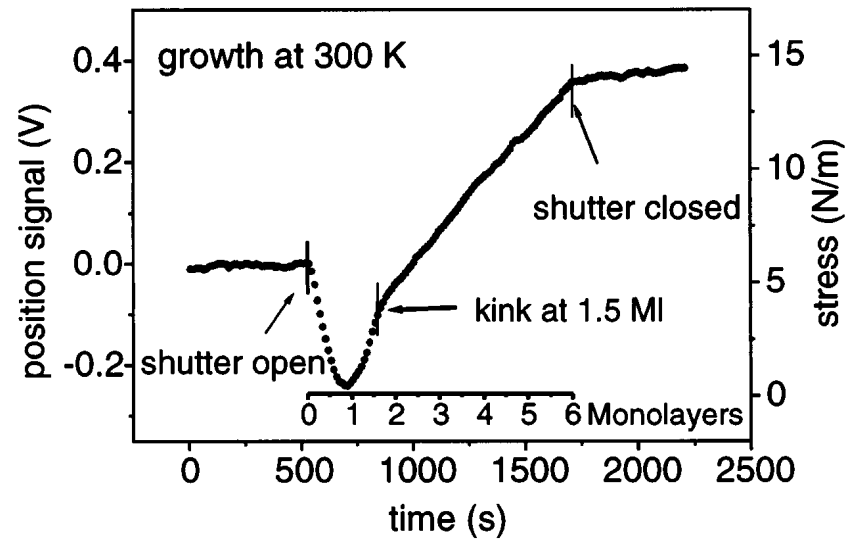

FIG. 1. Stress as a function of deposition time. From the growth rate of about $1 \AA / \mathrm{min}$, as checked by a quartz oscillator, the iron coverage has been deduced.

vs coverage curve. The kink in the stress curve lies at exactly that $1.5 \mathrm{ML}$ coverage where the beginning of the formation of misfit dislocations in the iron film is observed by STM. Figures 2(a) and 2(b) show STM pictures taken at coverages of 1.4 and 1.9 ML, respectively. Obviously, the most striking change in the STM pictures is that the added iron does not only enlarge the second layer islands, but also creates new nucleation sites on top of the islands. In accordance with a recent STM study by Bethge et al. [4] we ascribe those nucleation sites to the formation of misfit dislocations in the second layer patches; the kink in the stress curve indicates this change in the growth mode. From a coverage of $1.5 \mathrm{ML}$ on, misfit dislocations are formed that lead to a iron growth with a considerably decreased stress. In contrast to a stress study on the growth of $\mathrm{Ge}$ on $\mathrm{Si}$ [12], we observe a complete stress relief due to the change of the growth mode only for higher substrate temperatures around $1000 \mathrm{~K}$.

We assign the compressive stress found for small coverages to a true surface stress effect of the tungsten substrate. At this low coverage iron should be treated as an adsorbate, and it is well known from experiments that adsorbate induced surface stress is an important stress contribution [13]. Theoretical work indicates that even clean metal surfaces are under considerable surface stress [14]. The question whether iron actually induces compressive surface stress on $\mathrm{W}(110)$ or the adsorption of iron relieves tensile surface stress of the clean tungsten surface, giving rise to an apparent compressive stress, goes beyond the scope of this Letter.

The magnetism of the sesquilayer film at $1.5 \mathrm{ML}$ is characterized by an extremely enlarged coercive field in comparison to films of slightly lower and slightly higher thickness. In a narrow iron coverage range around $1.3 \pm$ $0.2 \mathrm{ML}$, the coercive field is increased by more than a factor of 10 (Fig. 3). This maximum of the coercive field as a function of the iron film thickness is most obviously seen in a MOKE experiment we performed on a film with a mesalike thickness variation. As shown in Fig. 3, our MOKE measurements at $140 \mathrm{~K}$ show a clear maximum of
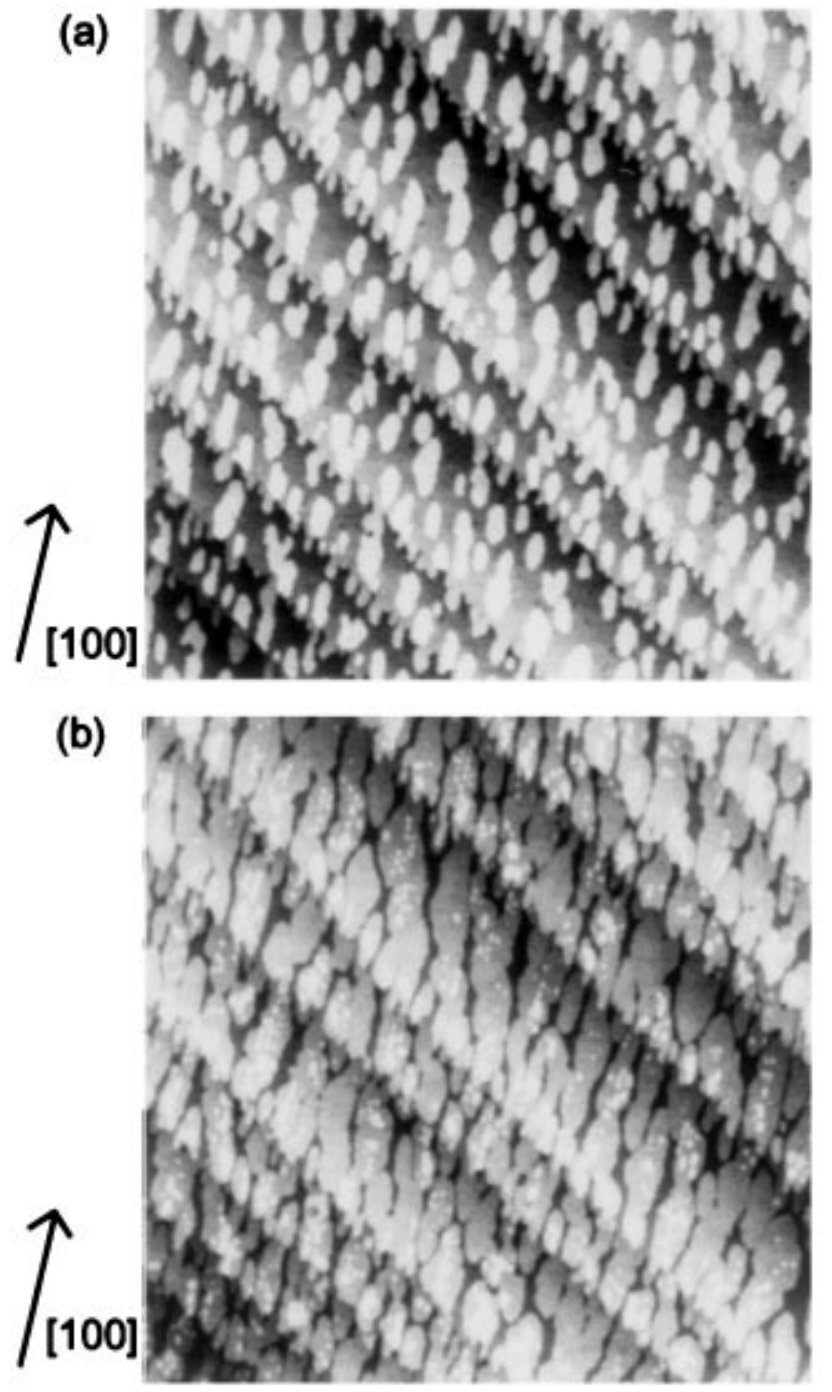

FIG. 2. STM images of iron on W(110). (a) $1.4 \mathrm{ML} F$ grown at $300 \mathrm{~K}$. Patches of the second layer on top of the first monolayer are visible. (b) The same area with a coverage of 1.9 ML. For both images the image size is $250 \mathrm{~nm} \times 250 \mathrm{~nm}$.

the coercive field at a coverage between 1.1 and 1.5 ML. Doing MOKE on a constant thickness film of 1.5 ML gives a coercive field larger than $0.3 \mathrm{~T}$ at $140 \mathrm{~K}$; thus, we cannot see hysteresis loops with our maximum magnetic fields at such low temperatures. At a slightly higher sample temperature of $195 \mathrm{~K}$ the coercive field has decreased to $0.2 \mathrm{~T}$, as shown in Fig. 3, curve (2).

Generally, nucleation of domains with reversed magnetization and pinning of domain walls are considered to be the main coercivity mechanisms $[15,16]$. However, the low coercivity of the 1 and 2 ML films indicates the presence of nucleation centers associated with a small number of imperfections in the film or at the film boundary; thus, we attribute the high coercivity to strong domain wall pinning.

The lowest-order anisotropy energy density can be written as [17]

$\eta_{a}=K_{1} \sin ^{2} \theta-\frac{1}{2} \mu_{0} M_{S}^{2} \sin ^{2} \theta+K_{P} \sin ^{2} \theta \cos 2 \phi$, 

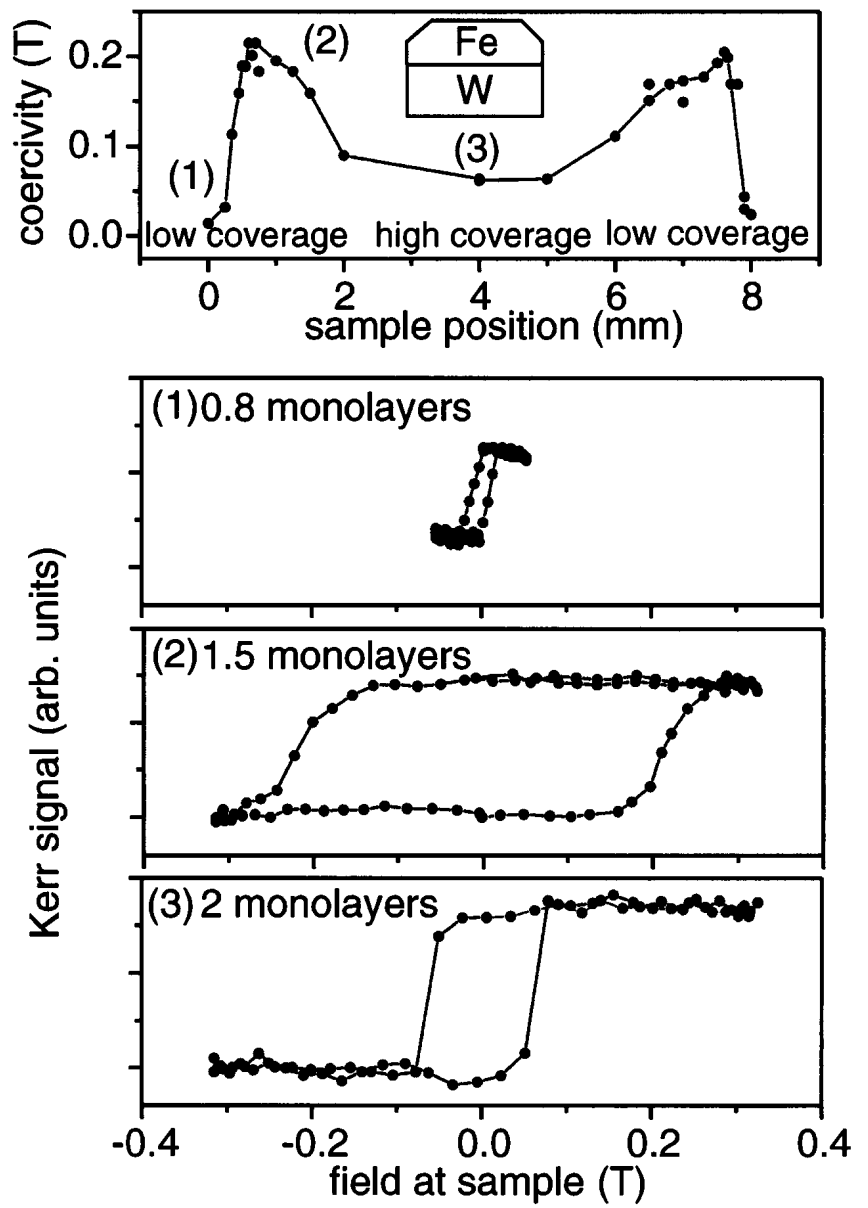

FIG. 3. Coercivity and hysteresis loops of iron films on $\mathrm{W}(110)$. The top graph shows the coercivity vs sample position obtained by spatially resolved MOKE measurements performed on a mesa shaped film at $140 \mathrm{~K}$. The iron film thickness increases from $0.8 \mathrm{ML}$ at one end to $2 \mathrm{ML}$ at the plateau and decreases down to $0.8 \mathrm{ML}$ again at the other end of the $\mathrm{W}(110)$ crystal. The solid curve serves as a guide to the eye. The labels (1) to (3) indicate the thicknesses as depicted in the hysteresis loops of the lower graphs. Curves (1) to (3) were obtained on films with a constant thickness. All films were deposited at $300 \mathrm{~K}$.

where $K_{1}$ and $K_{P}$ are the first uniaxial and in-plane anisotropy constants, respectively. The film normal is given by $\theta=0$. Higher-order anisotropy constants, such as $K_{2}$ [18], dominate the micromagnetic behavior if competing anisotropy contributions cause $K_{1}$ and $K_{P}$ to be very small. Ultrathin iron films on $\mathrm{W}(110)$ exhibit stable in-plane magnetization, $K_{1}<0$, so that higher-order anisotropy constants are negligible in lowest order. Since $K_{P}$ is positive, the preferred in-plane magnetization direction lies along [-110]. Minimizing Eq. (1) with respect to $\theta$ and incorporating the exchange and Zeeman energy densities $A(\nabla \phi)^{2}$ and $-\mu_{0} M_{S} H_{P} \sin \phi$, respectively, yields the micromagnetic free energy

$$
\begin{aligned}
E=\int[ & A(\nabla \phi)^{2}-2 K_{P} \sin ^{2} \phi \\
& \left.-\mu_{0} M_{S} H_{P} \sin \phi\right] d x d y d z .
\end{aligned}
$$

Here $H_{P}$ is the magnitude of the external magnetic field, which points in the easy in-plane direction $\boldsymbol{e}_{x}$. The exchange stiffness $A$, the saturation magnetization $M_{S}$, and the in-plane anisotropy constant $K_{P}$ incorporate bulk and surface contributions. In fair approximation, $K_{P}$ is dominated by surface effects, whereas magnetization and exchange stiffness are only slightly smaller than the respective bulk values. Let us therefore write $K_{P}=K_{S} / t$ where $t$ is the layer thickness and $K_{S}$ denotes the inplane surface anisotropy arising from the noncubic atomic environment of bcc (110) surfaces.

A necessary condition for magnetic reversal is the mobility of the in-plane domain walls (Néel walls). Following the standard procedure for calculating domain wall energies $\gamma$ and widths $\delta[15,19]$ we obtain from Eq. (2)

$$
\begin{aligned}
& \gamma=4 \sqrt{2 K_{S} A t}, \\
& \delta=\pi \sqrt{A t / 2 K_{S}} .
\end{aligned}
$$

Taking the monolayer thickness $t_{1}=2.03 \AA$ and using the estimates $K_{S}=0.6 \mathrm{~mJ} / \mathrm{m}^{2}$ [20] and $A=10^{-11} \mathrm{~J} / \mathrm{m} \mathrm{[21]}$ we obtain the monolayer values $\gamma_{1}=0.62 \times 10^{-11} \mathrm{~J} / \mathrm{m}$ and $\delta_{1}=4.1 \mathrm{~nm}$. For two layers, the result is $\gamma_{2}=$ $0.88 \times 10^{-11} \mathrm{~J} / \mathrm{m}$ and $\delta_{2}=5.8 \mathrm{~nm}$. A consequence of Eq. (3) is the pinning of Néel walls by two-layer patches: Because of the larger wall energy in the two-layer region it is energetically unfavorable for the domain wall to enter the island regions (Fig. 4). A rather unique feature of these equations is that the domain wall pinning originates from exchange inhomogenities, whereas pinning in typical permanent magnets is caused by anisotropy inhomogenities.

To estimate the maximum pinning force we consider the spatial dependence of the wall energy per unit length [15] $E_{\mathrm{W}}=\gamma(x)-2 \mu_{0} M_{S} H t(x) x$. Writing the average thickness as $t(x)=\left(1+x / x_{0}\right) t_{1}$, where $x_{0}$ is the range of inhomogenity, yields the small- $x$ energy derivative $\partial E_{\mathrm{W}} / \partial x=\gamma_{1} / 2 x_{0}-2 \mu_{0} M_{S} t_{1} H$. This means that external fields have to be larger than the coercivity

$$
H_{C}=\gamma_{1} / 4 \mu_{0} M_{S} t_{1} x_{0}
$$

in order to make the wall movement energetically favorable.

The maximum coercivity can be estimated by replacing $x_{0}$ by $\delta[15]$

$$
H_{C} \leq 2 K_{S} / \pi \mu_{0} M_{S} t_{1} .
$$

With the approximate magnetization $\mu_{0} M_{S}=2 \mathrm{~T}$ this equation yields the maximum coercivity $\mu_{0} H_{C}=0.59 \mathrm{~T}$. Note that the size of the patches, $5-10 \mathrm{~nm}$, as shown in Fig. 2(a), is comparable to the Néel wall width, so that the observed coercivity is well explained by Eqs. (5) and (6).

Another contribution to the increased coercivity originates from the inhomogenous stress [15] in the iron film. Our stress measurements suggest a maximum spatial variation of the film stress at a coverage around 1.5 ML, where the stress-driven formation of the misfit dislocations starts. From the slope of our stress curve, this stress variation can 
(a)

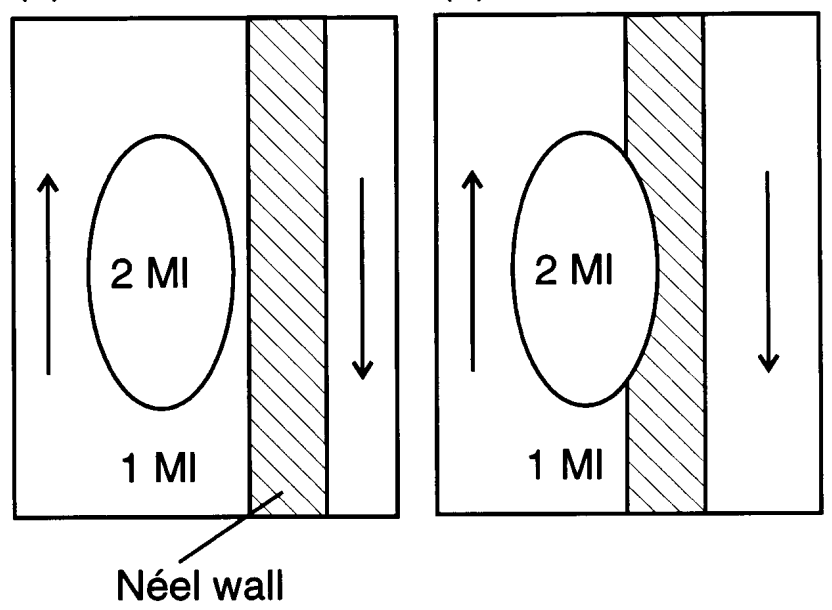

FIG. 4. Pinning of a Néel wall (dashed area) in an ultrathin film. (a) Mobile wall in a monolayer region and (b) pinning at a second layer island. The arrows indicate the opposite magnetization of the adjacent domains.

be estimated to be of the order of $50 \%$ of $4 \mathrm{~N} / \mathrm{m}$ per monolayer $\mathrm{Fe}$, or $10 \mathrm{GPa}$, corresponding to a strain parameter of roughly $5 \%$. This value has to be compared to the nominal strain $\sqrt{2}-1=41 \%$ associated with the distorted environment of iron atoms in a 1 ML (110) film. Assuming a linearly increasing anisotropy with lattice deformation [16] this leads to a coercivity of order $0.07 \mathrm{~T}$. Thus, the stress contribution is much smaller than the crystalline one, but not necessarily negligible.

The main pinning mechanism as shown in Fig. 4 is rather unique in the sense that the energy difference between the different regions is an exchange effect: Entering the $2 \mathrm{ML}$ region is energetically unfavorable due to the enhanced domain wall exchange energy. By comparison, pinning in permanent magnets largely originates from anisotropy inhomogenities [15,16]. In fact, Eq. (3) shows that the dilution of the surface anisotropy has actually the wrong sign but is overcompensated by the increased exchange, i.e., by the increased number of iron-iron bonds in the 2 ML patches. Finally, note that this behavior is restricted to (110) layers [7]: $K_{S}$ in Eq. (6) equals zero for (100) and (111) surfaces. By analogy, we can expect that third layer islands on a 2 ML film reintroduce some pinning, thus leading to a coercivity minimum for $2 \mathrm{ML}$. However, Eq. (3) shows that the difference in domain wall energy is largest between films of 1 and 2 ML thickness. This means that the amplitude of the coercivity oscillations will decrease drastically with increasing layer thickness.

In conclusion, our stress measurements clearly show how the misfit induced film stress is lowered by the formation of misfit dislocations. We have shown that sesquilayer iron films on W(110) exhibit pinning-type domain wall freezing rather than quasiantiferromagnetic exchange as suggested in [8]. The main evidence comes from coercivity data, which are explained in terms of domain wall pinning at 2 ML regions, where the exchange energy of the walls is higher than in $1 \mathrm{ML}$ regions. A prediction of the model is coercivity oscillations whose amplitude decreases strongly with increasing layer thickness.

[1] Crystal Data, edited by J.D. H. Donnay and H. M. Ondik (National Bureau of Standards, Gaithersburg, MD, 1973).

[2] Throughout this Letter we define $1 \mathrm{ML}$ as the pseudomorphic monolayer which is characterized by a coverage of one iron atom per W(110)-surface atom (1 monolayer $=$ $1 \mathrm{ML}=1.41 \times 10^{15} \mathrm{Fe}$ atoms $\left./ \mathrm{cm}^{2}\right)$.

[3] M. Przybylski, I. Kaufmann, and U. Gradmann, Phys. Rev. B 40, 8631 (1989).

[4] H. Bethge, D. Heuer, Ch. Jensen, K. Reshöft, and U. Köhler, Surf. Sci. 331-333, 878 (1995).

[5] Nearest neighbor interaction $f=3 / 2 a c_{44}$, next nearest neighbor interaction $f^{\prime}=1 / 2 a\left(c_{11}-c_{12}\right)$, G. Leibfried, Handbuch der Physik, edited by S. Flügge (Springer, Berlin, 1955), Band VII/1, p. 201.

[6] H. J. Elmers, J. Hausschild, H. Höche, U. Gradmann, H. Bethge, D. Heuer, and U. Köhler, Phys. Rev. Lett. 73, 898 (1994).

[7] H. J. Elmers and U. Gradmann, Appl. Phys. A 51, 255 (1990).

[8] H. J. Elmers, J. Hausschild, H. Fritzsche, G. Liu, U. Gradmann, and U. Köhler, Phys. Rev. Lett. 75, 2031 (1995).

[9] D. Sander, A. Enders, and J. Kirschner, Rev. Sci. Instrum. 66, 4734 (1995).

[10] S. D. Bader, J. Magn. Magn. Mater. 100, 440 (1991).

[11] Ch. Kittel, Introduction to Solid State Physics (Wiley, New York, 1986), 6th ed., p. 560.

[12] A. J. Shell-Sorokin and R. M. Tromp, Phys. Rev. Lett. 64, 1039 (1990).

[13] D. Sander, U. Linke, and H. Ibach, Surf. Sci. 272, 318 (1992).

[14] V. Fiorentini, M. Methfessel, and M. Scheffler, Phys. Rev. Lett. 71, 1051 (1993).

[15] E. Kneller, Ferromagnetismus (Springer, Berlin, 1962).

[16] R. Skomski and J. M. D. Coey, Phys. Rev. B 48, 15812 (1993).

[17] The factor $\frac{1}{2}$ in the magnetostatic energy is the perpendicular demagnetizing factor in SI units.

[18] D. J. Craik and R. S. Tebble, Rep. Prog. Phys. 24, 116 (1961).

[19] S. Chikazumi, Physics of Magnetism (Wiley, New York, 1964).

[20] H.J. Elmers and U. Gradmann, Appl. Phys. A 51, 255 (1990). Note that anisotropy constants tend to increase with decreasing temperature, which explains the even higher coercivity below $140 \mathrm{~K}$.

[21] R. Skomski et al., Interstitial Intermetallic Alloys, edited by F. Grandjean and K.H.J. Buschow (Kluwer, Dordrecht, 1995). 